\title{
Reoperative surgery in anorectal malformation patients
}

\author{
Timothy F. Tirrell ${ }^{1}$, Erin R. McNamara ${ }^{2}$, Belinda H. Dickie ${ }^{1}$ \\ ${ }^{1}$ Department of Surgery, ${ }^{2}$ Department of Urology, Boston Children's Hospital, Boston, MA, USA \\ Contributions: (I) Conception and design: BH Dickie; (II) Administrative support: None; (III) Provision of study materials or patients: None; (IV) \\ Collection and assembly of data: ER McNamara; BH Dickie; (V) Data analysis and interpretation: All authors; (VI) Manuscript writing: All authors; \\ (VII) Final approval of manuscript: All authors. \\ Correspondence to: Belinda Dickie, MD, PhD. Department of Surgery, Boston Children's Hospital, 300 Longwood Ave, Fegan 3, Boston, MA 02115, \\ USA. Email: Belinda.dickie@childrens.harvard.edu.
}

\begin{abstract}
Currently accepted primary repair of congenital anorectal malformations (ARMs) includes a posterior sagittal incision, which allows for optimal visualization and identification of important pelvic structures and anatomical features. Reconstructive surgery involves meticulous dissection and separation of pelvic structures, and careful reconstruction can result in good functional outcomes for many patients, who live without ongoing sequelae from their malformation. However, some patients may require reoperative procedures for anatomic or functional reasons. Males and females present with slightly different symptoms and should be approached differently. Males are most likely to require reoperations for anorectal or urethral pathologies, but the urinary system is often spared in females-they instead must contend with Mullerian duct anomalies, of which there are many varieties. Depending on the original malformation and severity of symptoms, redo surgery may be needed to optimize function and quality of life. Surgical management with reoperative surgery in ARMs ranges from straightforward to complex, depending on the issue. One must weigh the risks of reoperative surgery and potentially creating more scarring against the need for a better anatomical and functional outcome. Current management trends and practice patterns with regards to reoperative surgery in ARM patients are not widely studied or standardized but we provide an overview of the more common pathologies, preoperative evaluation and workup required to identify the issues, and options for reoperative repair in these patients.
\end{abstract}

Keywords: Anorectal malformation (ARM); reoperative; redo surgery

Received: 05 May 2020; Accepted: 24 June 2020; Published: 25 July 2021.

doi: $10.21037 /$ tgh-20-214

View this article at: http://dx.doi.org/10.21037/tgh-20-214

\section{Introduction}

Surgical treatments for anorectal malformations (ARM) have been described for centuries, with varying success. In the 1700s and 1800s surgical repair of imperforate anus included blind exploration of the perineum to find the rectum. It was not until the late 1800 s that a procedure to identify the rectum from above was described if it could not be found from below. With the advent of radiography, anorectal anomalies could be better defined and locating the rectum prior to surgical exploration became possible. In the 1950's Stephens described the importance of the puborectalis muscle (1) and the need for preservation of the musculature and sphincter complex in describing his technique for optimizing functional outcome (2). Prior to the 1980s, the most common approach to ARM was the abdominoperineal approach. After the posterior sagittal anorectoplasty (PSARP) was described by deVries and Peña in $1982(3,4)$, it was soon adopted as the standard operative repair and remains so, having withstood the test of time. With their approach, the sphincter muscle complex, perineum, and pelvic structures are reliably exposed to allow for an optimal anatomic repair. Broad exposure via a midline posterior sagittal incision allows full rectal mobilization while minimizing the risk to surrounding structures. Precise identification of the sphincter complex 
allows for equal division, which facilitates exact placement of the neoanus in the middle of the sphincter complex. This approach was seen as imperative in correcting ARM. Shortly after this description, the technique was applied to patients who had undergone prior ARM repair and needed a revision or complete redo operation (5). Georgeson et $a l$. applied a laparoscopic approach to repair of ARM and reported his series of laparoscopic rectal mobilizations and fistula divisions with perineal pull-through (6). There is no universally applicable optimal operative approach, as it is dependent on multiple factors including fistula level, sacrococcygeal anatomy, associated malformations, and surgeon experience and preference.

Regardless of approach, many children with ARM undergo an uneventful repair without significant longterm issues. Although functional stooling and voiding challenges are not uncommon, many "low-type" ARMs such as rectoperineal and rectovestibular fistulas achieve continence outcomes and report quality-of-life scores consistent with control patients at mid- $(7,8)$ and longterm (9) time points. However, many are not so fortunate (10). Some common symptoms can be managed medically but these patients merit evaluation for potential benefit of reoperative intervention to optimize functional outcome. Some patients may require repeat operations for anatomic problems that arise as technical complications from surgery or from unrecognized diagnoses.

\section{Complications after primary repair}

Complications after ARM repair are well documented but are not necessarily indications for reoperation. Severity of symptoms is an important consideration as not all complications cause morbidity to the patient and may not need surgical correction. Among symptomatic patients, common indications for repeat operations can be categorized into anatomic and functional issues, and some are specific to males or females.

Common anatomic issues in males are usually related to rectal or urethral problems. Anorectal stricture, mislocation, rectal prolapse, and wound dehiscence are all problems related to the rectum. Patients whose original malformation included a long perineal fistula or a rectourethral fistula are at risk for urethral problems, including urethral stricture, persistent fistula (if the original fistula was not recognized or if the urethra was damaged), and posterior urethral diverticulum (PUD), also known as remnant of the original fistula (ROOF).
Females are at risk for similar rectal complications, but analogous urethral problems are usually only seen in patients with cloacal malformations. Mullerian anomalies, including retained vaginal septum, undrained hydrocolpos (or hydrometra or hydrometrocolpos), absent vagina, and introital stenosis are identified in this population. Patients with cloacal malformations are at risk for persistent urogenital (UG) sinus secondary to surgical technique or as a result of a missed diagnosis, having been originally incorrectly classified as a rectovaginal fistula.

Functional issues common to both genders include fecal incontinence, constipation, and overflow pseudoincontinence.

The true incidence of these complications is not well characterized as most existing data are generated from retrospective cohort review and sample sizes are small. Understanding the frequency of outcomes is further clouded by the lack of standard reporting scales. Functional scales [such as the bowel function score described by Rintala (11), the Baylor continence scale (8)] and objectively collected data [such as anorectal manometry (12)] are common but not universally reported. Many different tools for describing outcomes are found in the literature, complicating comparisons between studies. Other functional domains such as social problems, quality of life, sexual function, and fertility have been investigated (9), but are even less standardized in their reporting tools. Interpretation of these scores is confounded by a known relationship between severity of malformation and poorer functional outcomes.

\section{Workup of patients with anorectal anomalies after repair}

Presenting symptoms are variable, but often related to challenges with urinary or fecal continence. Although details of the initial operation may not always be known, a standard evaluation and assessment of the patient often yields information relevant to original anatomy and current issues. This challenge was discussed in a recent article by Lane et al. (13), wherein the authors detailed their manner of assessment of internationally adopted children, many of whom present with incomplete medical records. $77 \%$ of the population reviewed ultimately underwent reoperative surgery, suggesting a significant need for corrective operations in this population.

Detailed physical examination (which may require anesthesia), endoscopic procedures (cystoscopy, vaginoscopy) and stimulation of the anal sphincter complex 
are generally the most important parts of the evaluation. They provide information about anatomical issues such as prolapse, anal mislocation, and wound problems, and may also facilitate identification of persistent fistulas. Examination may also demonstrate improper diagnosis of the original malformation. Levitt et al. (14) found a significant rate of persistent UG sinus in cloaca patients who were originally diagnosed with rectovaginal fistula. The series of patients summarized by Rosen et al. (15) suggests similar findings, and that true rectovaginal fistulas are quite rare, even among the ARM population. In both studies, many patients had undergone rectal separation and pull-through but were left with a persistent UG sinus due to incorrect diagnoses.

Choice of additional diagnostic studies depends on presenting symptoms and suspected pathologies. Voiding cystourethrogram or contrast enhanced cystography (16) are useful for evaluating the lower urinary tract. Urinary complaints may also be secondary to neurologic issues, so spinal MRI to assess for spinal malformation (most commonly tethered cord) and urodynamic studies may be considered to help differentiate underlying causes as anatomic or neurologic. Plain film radiography may help evaluate stool burden and differentiate fecal incontinence from pseudoincontinence. Anorectal manometry and colonic motility studies may help estimate the degree to which motility issues of the colon contribute to the symptoms at hand.

\section{Indications for redo operations}

No clear evidence-based or consensus guidelines exist regarding indications for repeat operation in ARM patients. Groups reporting their indications in the context of research studies demonstrate a broad variety. Rentea et al. (17) laid out indications for ROOF repair, which included UTI, dribbling, mucus via urethra, stone formation, or needing a smooth posterior urethra for clean intermittent catheterization. Ardelean et al. (18) formally laid out guidelines for reoperation for incontinence as failure of conservative therapy. Initial therapy included bowel management and dietary control for modification of stool consistency, but criteria for failure of therapy were not strictly controlled. In a patient series described by Peña $e t$ al. (19), indications for surgery differed based on presenting symptoms-describing that patients with fecal incontinence after PSARP will clearly have different indications than those referred after "catastrophic complication" following their initial operation. Hrabovszky and Dewan (20) rarely used imaging or functional studies to determine operative candidacy, instead utilizing gross changes notable on examination in conjunction with an appropriate patient history. Although the specifics vary across studies, a unifying theme appears to be that findings suggestive of anatomic or functional issues, in the presence of a concordant patient history, encourage surgeons to offer repair.

\section{Rectal indications}

For both males and females, issues related to the rectum are likely the most common issues to result after repair of imperforate anus.

\section{Mislocation}

During initial repair, the sphincter muscle complex is identified and placement of the anoplasty must be centered in both the anterior-posterior and left-right planes. While theoretically uncomplicated, difficulties often arise with precise placement and anal mislocation can compromise functional outcome. A combination of stimulation and direct visualization of the muscles during the deepening of the posterior sagittal incision enhances identification of the middle of the sphincter complex, facilitating accurate placement of the neoanus. Anal mislocation is unfortunately common despite these precautions (Figure 1) but can be corrected with a redo anoplasty.

Circumferential dissection around the rectum is performed, proceeding proximally as needed for adequate mobilization. By staying along the bowel wall, it can safely be separated from the sphincter muscle complex without damaging or removing the existing muscle complex. The rectum is mobilized proximally until it can be moved safely without tension to the center of the muscle complex. Anterior-posterior mislocation can be easier to correct than lateral mislocation but the principles remain the same; with careful dissection the rectum is mobilized and then fixed in place in the center of the muscle complex. However to address lateral mislocation, the muscle complex must be split more in the middle and the neoanus tunneled over.

\section{Stricture}

Strictures are often found at the level of the skin but can also extend proximally. Skin-level strictures (Figure 2) can be addressed with a Heineke-Mikulicz type of stricturoplasty. 


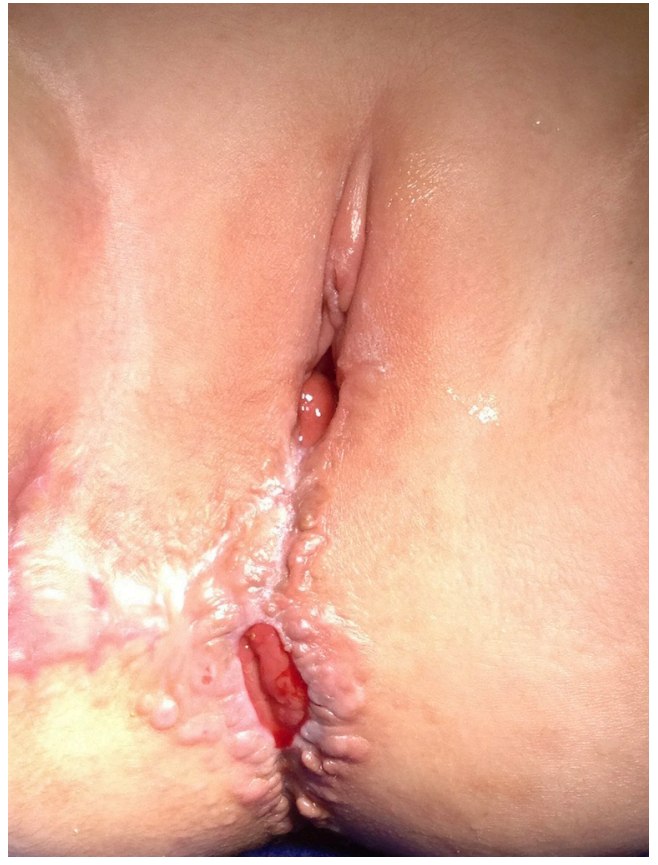

Figure 1 Prolapse and posterior mislocation of the neoanus. The neoanus is located posterior to the perineal dimple that likely corresponds the location of the sphincter complex.

Multiple radial incisions across the anocutaneous junction are made and then repaired transversely, allowing the anoplasty to enlarge to a sufficient size (21). This can be performed as an outpatient operation without preoperative bowel preparation, and can be highly effective in treating short, skin-level strictures.

Longer strictures are thought to arise from tension or ischemia of the rectum at the time of the initial surgery. They can also result from postoperative wound infections and dehiscence of the anoplasty. Symptoms that may alert a clinician to evaluate for longer or more proximal strictures include painful dilations or difficulty stooling. Diagnosis is confirmed by a combination of an examination and contrast enema. The strategy for addressing the stricture is somewhat dependent on location and length. To address these longer strictures, pull-through of more proximal healthy rectum is usually required. Full mobilization of the rectum is needed to bring down healthy bowel to the perineum, which may require intraabdominal mobilization of the colon. It is important to note that if the bowel to be brought down to the perineum is no longer rectum, intramural blood supply may not be sufficient to supply the bowel and the dissection must preserve the vascular

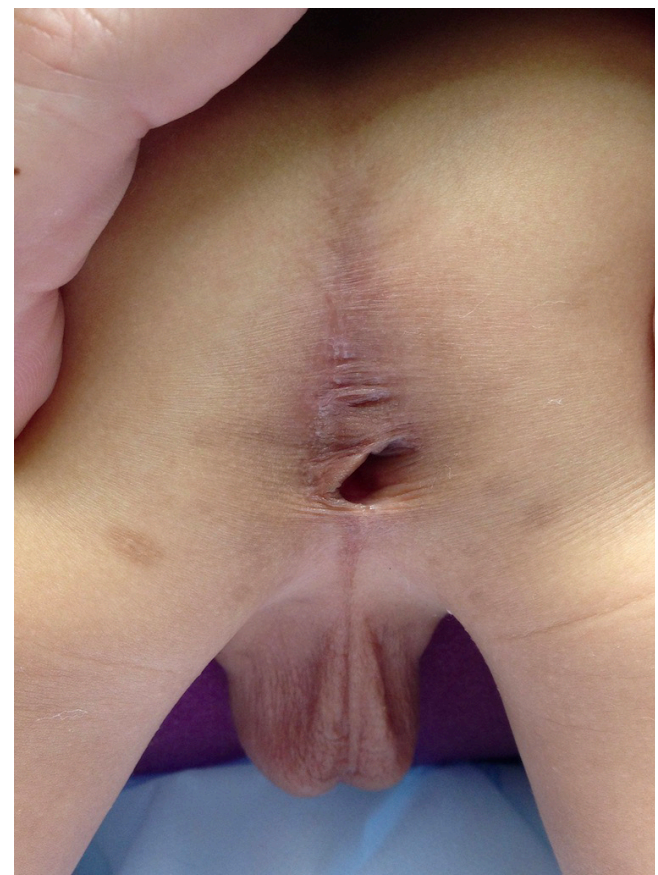

Figure 2 Neoanal stricture and anterolateral mislocation. The stricture would be readily corrected with a Heineke-Mikulicz type stricturoplasty but this patient also requires correction of his mislocation.

pedicle. Strictures may also occur at the level of the pelvic floor. Examination under anesthesia will demonstrate a stricture higher and is usually felt on digital rectal examination or with resistance to passing dilator. The distal rectum and anoplasty usually remain supple. These strictures are thought to occur secondary to inadequate opening or dilation of the levators during the initial pullthrough [especially if performed laparoscopically (22)], or due to scarring of the levators during the reconstruction. If no stricture is detected on exam under anesthesia but the patient presents with obstructive-type symptoms, an index of suspicion should be maintained for this pathology. Contrast enema is useful for diagnosing strictures more proximal than can be detected on digital examination. This issue can be addressed with a posterior sagittal incision, though which the levator and pelvic floor space are opened while leaving the anoplasty intact.

\section{Prolapse}

Anorectal prolapse can be a common problem after anoplasty and the frequency is higher in patients with 


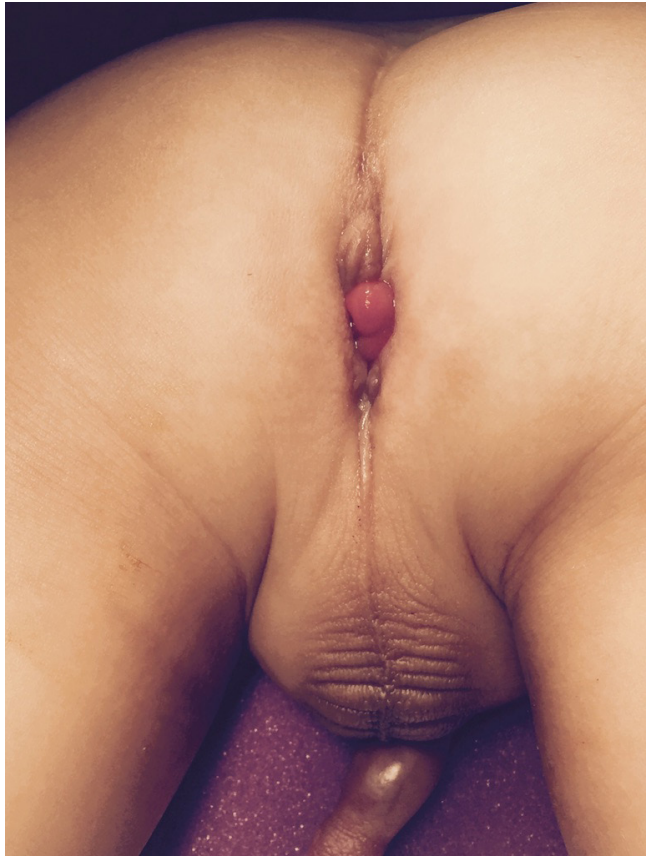

Figure 3 Rectal prolapse. The prolapse is not fully circumferential and can therefore be managed with a local excision.

poorly developed musculature at the perineum (higher malformations or longer common channels). It also is more common with children who strain with stooling due to uncontrolled constipation (23). Repair of prolapse is indicted if the area is symptomatic-most common symptoms include mucus production, bleeding, and discomfort. It is also indicated if the child has issues with stool leakage or is close to having bowel control but is unable to completely close the anal opening due to the prolapsed mucosa. Prolapse repair is usually performed transanally and involves circumferentially excising excess tissue and repairing the bowel-skin anastomosis. It often helps to trim the bowel mucosa somewhat shorter to help prevent future prolapse. Some children only prolapse one side of their neoanus (Figure 3); this can also be addressed by excising the affected side to match the non-prolapsed side. Parents should be advised that operative repair of prolapse addresses symptoms only and that the risk of recurrence can be high (24), especially if the child continues to strain.

\section{Retained dilated segment}

For children with difficulties achieving fecal continence, plain film radiography can be used to evaluate stool burden and help differentiate incontinence from overflow pseudoincontinence. If symptoms are refractory after enrollment in a bowel management program, manometry and motility evaluation may be useful in establishing the function of the bowel. Many patients are found to have significantly dilated portions of their colon/rectum at reoperation, suggesting that disordered motility and function may contribute to their symptoms (20). In these patients, a limited resection or tapering of the dilated segment of colon may aid in improving functional outcomes. Strong data and firm guidelines that may guide the choice of the optimal operation are absent. As such, the decision to taper or resect dilated colon is largely based on the anticipated residual function of the segment, which can be estimated though manometry and motility testing. If dysmotile, the patient is likely best served by a resection. If motility is somewhat preserved or the colon is very short, tapering may be a more appropriate initial operation, as the segment can later be resected if it proves to be completely nonfunctional.

\section{Urinary tract indications}

Urinary tract complications are more common in males than females as the rectum is intimately apposed with the urethra, requiring separation and division of a common wall during the initial anorectoplasty.

\section{Urethral stricture}

The dissection of the rectal fistula requires careful attention to mitigate risk of damage to the urethra. The common wall between the rectum and urethra must be separated carefully as this dissection raises the potential for urethral damage or ischemia. The ischemia and resultant stricture may not be detected immediately but a high index of suspicion must be maintained in postoperative patients who present with symptoms such as recurrent UTI, difficulty placing a foley catheter, or imaging evidence of voiding dysfunction (enlarged bladder or hydronephrosis). Strictures can sometimes be managed with cystoscopy and dilation or incision of the stricture but if severe, may need to be addressed with a formal urethroplasty. Due to the complexity of repair and the reoperative environment, we recommend that this be performed as a combined procedure in collaboration with a pediatric urology team. Often the most straightforward approach is via the previous posterior sagittal incision. The rectum is mobilized to allow 


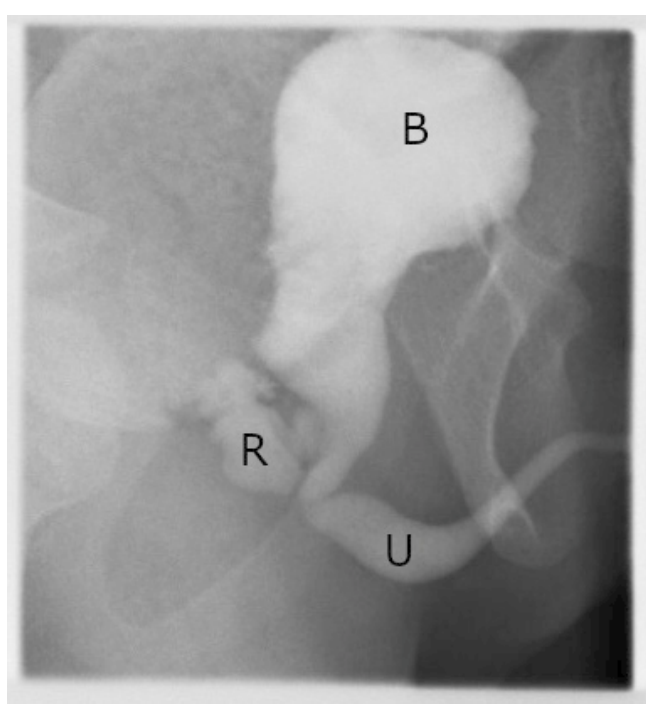

Figure 4 Voiding cystourethrogram demonstrating the presence of a posterior diverticulum/remnant of the original fistula. B, bladder; $\mathrm{U}$, urethra; $\mathrm{R}$, remnant of the original fistula.

direct access to the posterior urethra, where the stricture can be identified and repaired.

\section{Retained remnant of the rectal fistula}

Urinary symptoms [most commonly daytime dribbling (25) or urinary incontinence] should raise concern for a PUD or ROOF. As laparoscopic repair has become more popular for takedown of recto-urethral fistulas, some have expressed concern about possible association with increasing frequency of PUD/ROOF (25). Bladder neck and high prostatic fistulas are easily accessed laparoscopically, but distal fistulas have a longer common wall that must be separated off of the posterior urethra prior to division of the fistula. This division is challenging and poses risk to the urethra (see Urethral Stricture, above), likely leading some surgeons to terminate the dissection prematurely, prior to the actual entrance of the fistula. This complication underscores the importance of precisely defining the location of the fistula prior to the index operation-considered by some to be the most important information to be obtained in the preoperative evaluation (26). When evaluating a patient for a redo operation, a PUD/ROOF may be detected via cystoscopy (Figure 4), VCUG or sometimes pelvic MRI (27).

If the PUD/ROOF is small and asymptomatic, it may be observed but should undergo regular surveillance to ensure that it does not increase in size or become symptomatic. If the PUD/ROOF enlarges or is symptomatic, it can be approached via a redo posterior sagittal incision. As with a stricture repair, this incision allows mobilization of the rectum and direct access to the posterior urethra. Laparoscopic repair has been described (28), however, one must make sure not to make the same mistake as the initial surgery. Simultaneous cystoscopy to locate the fistula during the dissection, or placement of a stent into the fistula, may be helpful for identification of the PUD/ROOF and ensure complete removal.

There is also the theoretical risk of developing adenocarcinoma in the ROOF that is exposed to urine. While cases have been reported (29) there is no indication that this warrants removal or surveillance of an otherwise asymptomatic ROOF.

\section{Persistent/recurrent rectourethral fistula}

Although uncommon, this is usually a result of damage to the urethra and rectum during the original pull-through or misidentification of the fistula. Presenting symptoms include recurrent UTI, urine via the anoplasty, or sediment in the urine. As with the other urethral pathologies presented, optimal diagnostic tools include VCUG and cystoscopy, and newer imaging modalities such as contrast enhanced ultrasound may prove useful as well (30). A redo pull-through is necessary to completely separate the rectum from urethra and excise the fistula tract. Once separated, interposition of fat or muscle is recommended to help maintain separation between the two structures. In addition, healthy rectum with no violation of the wall should be pulled through to the perineum.

\section{Mullerian structure indications}

Up to $10 \%$ of female ARM patients have associated Mullerian anomalies, which include uterine (septate, bicornuate, unicornuate, didelphys) or vaginal (septate, complete absence, distal absence) malformations. The introitus should be assessed at the initial operation to evaluate for vaginal malformations. Timing of vaginal surgery is without clear consensus-while some suggest waiting until after puberty or when the patient is 


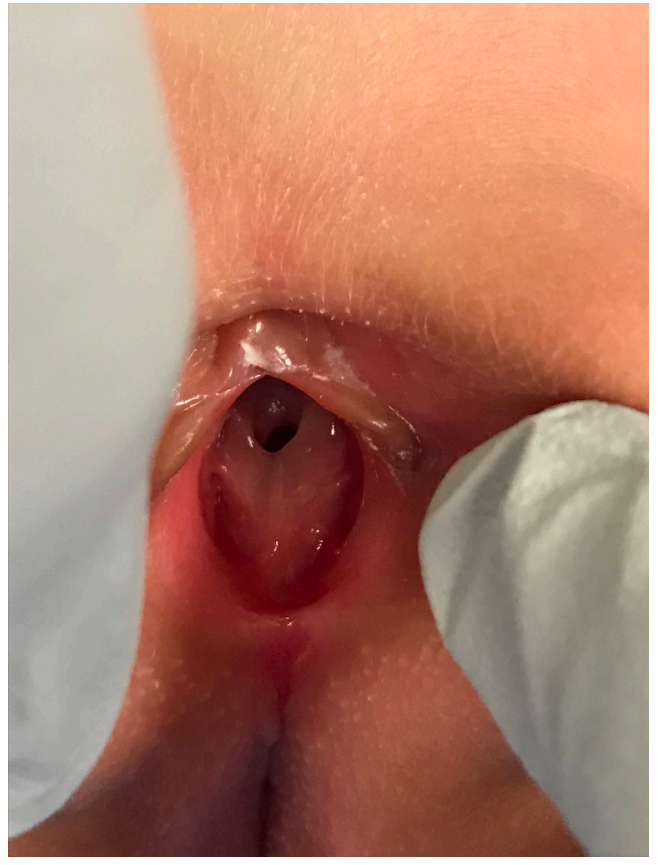

Figure 5 Absent vagina. This diagnosis is not appreciated without a close introital examination.

psychologically developed, others advocate for addressing the anatomic abnormalities when discovered.

\section{Longitudinal vaginal septum}

This is easily addressed via perineal approach. The septum does not cause functional or symptomatic difficulties if both hemi-vaginas are unobstructed, so operative timing is generally not critical. The septum is resected in a distalto-proximal direction and the proximal limit must be chosen so that it does not cause scarring around the cervix. We recommend performing vaginoscopy during the same procedure to define the nature of any associated uterine malformations, most specifically the presence of a vaginal septum or multiple cervices. A single cervical os is usually associated with normal upper uterine structure. However, there are some uterine anomalies that have a single external cervical os, so maintaining a level of suspicion for anomalies is important. One recent study of 63 female ARM patients found that longitudinal vaginal septum was the most frequently found vaginal anomaly, and all patients with vaginal anomalies had an associated uterine anomaly (31). Understanding if a patient is at elevated risk for uterine anomalies is important it may have obstetric implications.
For those in whom a longitudinal septum is found, septal division and oversewing the cut edges for hemostasis is a straightforward procedure.

\section{Absent vagina}

If the perineum is not fully examined at the initial operation, this diagnosis may be missed (Figure 5). Common presenting symptoms may include amenorrhea or pain. Cyclical abdominal or pelvic pain after the onset of puberty may be secondary to hydrometrocolpos, signaling the presence of an upper vagina and uterus. Fully defining the internal Mullerian structures is important before starting potential reconstruction. This can be performed via imaging (ultrasound and/or MRI) or through laparoscopic evaluation at the time of repair.

If the proximal vagina is present, restoration of continuity with distal vaginal tissue is optimal and can be achieved by joining the proximal vagina to the distal vagina. Proximal stretching of the introital area can facilitate elongation of the distal vaginal mucosa, and the pullthrough is accomplished through a combined abdominal and perineal approach. If the distance is too great and poses risk of vaginal devascularization, the surgeon should be prepared to utilize other methods to bridge the gap. Buccal or bowel grafts are most effective at accomplishing this, but many techniques of vaginal replacement are described in the literature and no consensus exists on the optimal method (32). We therefore recommend that any surgeons attempting repair of absent vagina should be familiar with multiple repair options, should the gap between proximal and distal vaginal segments prove too large for primary repair. If the upper vagina and uterus are absent, reconstruction can be performed utilizing the similar tools, including stretching of the distal vagina, buccal grafts, or bowel grafts. It is important to note that although bowel may be a readily available graft source, it is not without its own complications, including stenosis, prolapse, discharge, and other uncommon but significant challenges such as inflammatory bowel disease and neoplasms.

One rare finding is a missed hemivagina that is not patent to the introitus and perineum. These patients will present with abdominal or pelvic pain after the onset of puberty but can have regular menstrual cycles as one hemivagina is patent to the perineum. Examination may demonstrate a palpable mass, and imaging will show a dilated Mullerian structure (Figure 6). If the septum cannot be reached or divided from below to drain the vagina, a 


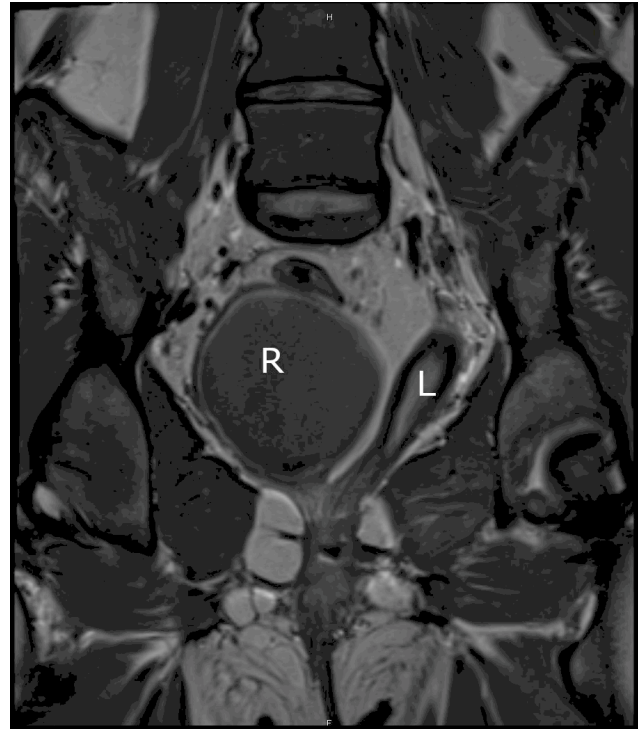

Figure 6 Coronal section of pelvic MRI demonstrating duplicated vagina with right sided vaginal obstruction. The right hemivagina $(\mathrm{R})$ is significantly dilated and the left hemi-vagina $(\mathrm{L})$ is nonobstructed and displaced leftwards.

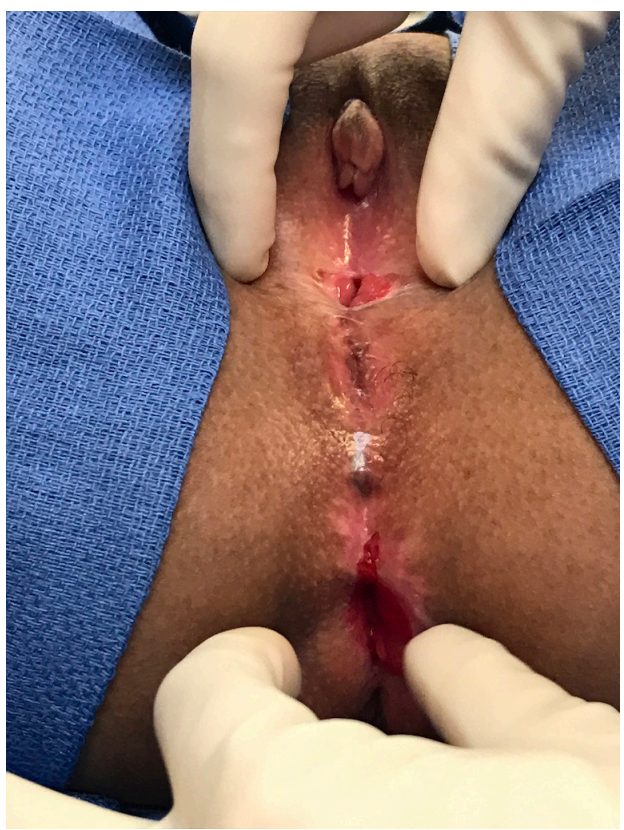

Figure 7 Vaginal stenosis and mislocation and rectal posterior mislocation. This patient requires a repeat posterior sagittal anorectovaginoplasty to adequately mobilize the vagina and rectum to allow placement in its anatomic location. hemi-hysterectomy may be required. If so, care should be taken to preserve the ipsilateral ovary and contralateral uterus, vagina, and ovary.

\section{Introital stenosis}

The introitus should be assessed for adequacy prior to puberty and then prior to intercourse to ensure an adequate opening size. If stenosis is skin-level (Figure 7), a stricturoplasty can be performed, similar to that described for anal stricture. If deeper than skin level, vaginoplasty with a buccal mucosal graft can be performed. Dilations are frequently required after these procedures so patient readiness is an important preoperative consideration.

\section{Rectovaginal fistula}

Similar to recurrent rectourethral fistula, these are uncommon but usually a result of damage to the posterior vaginal wall and rectum that occurred during the initial operation. This fistula is usually symptomatic, with stool or gas passing out the vagina. It can be defined on clinical examination, which should also include vaginoscopy and sigmoidoscopy. MRI may also prove useful in helping define fistulas that are more complex or challenging to identify. For repair, a posterior sagittal incision is performed and the rectum is mobilized. Complete separation of the rectum from the vagina may be required to address the fistula. Local repair with a rectal advancement flap is possible, but it is important that the resultant suture lines not cross as this is thought to place the patient at higher risk for recurrence (33). As with the rectourethral fistula repair, interposition of fat or muscle helps separate the two structures and mitigate this risk as well. Further, healthy and undamaged rectum wall should be opposed against the posterior vaginal wall to ensure adequate healing and repair of the fistula.

\section{Persistent urogenital sinus}

A persistent UG sinus is specific to cloaca repairs. It generally results from initial misdiagnosis $(14,15)$ or inadequately addressing the urethra and vagina during the cloaca repair. Repair of the UG sinus should be performed prior to menarche to ensure an adequate outflow tract for 
menstrual products.

Surgical management of UG sinus depends on the anatomy of the relevant structures. Contrast studies and endoscopy are useful for defining the length of the common channel and location of the vagina in relation to the bladder neck, which are relevant parameters for choosing surgical approach. The two primary repair options are UG mobilization and UG separation. For previously unrepaired patients, the choice of mobilization or separation is largely dependent on the lengths of the common channel and urethra, as common channel length may dictate if abdominal dissection is needed and urethral length may play a role in ability to achieve postoperative urinary continence (34). For those patients whose persistent UG sinus is secondary to inadequate initial UG mobilization, repeat UG mobilization should be weighed against UG separation. The optimal approach depends on urethral anatomy, the mobility of the vagina, and the experience of the surgeon (35). It should be noted that both primary and redo UG mobilization carry significant risk of urinary incontinence in (36).

\section{Outcomes from reoperative interventions}

Most studies in the literature that describe outcomes from repeat operations in ARM patients report symptomatic improvement for the majority of patients treated. However, studies are usually uncontrolled and sample sizes are generally small, owing to the low incidence of ARM. Outcome measures are disparate, making direct comparisons or pooled statistics challenging to interpret. Similar to measures used after primary surgery, some studies report outcomes with standardized scales such as Rintala scores, and some use self-designed outcome surveys that have high risk for bias.

Although some ARM patients undergo a PSARP and do not have long term sequelae, it is clear that many encounter significant challenges over their lifetime and some of these challenges are related to their original surgery. Therefore, despite the lack of high-quality, prospective literature demonstrating clear efficacy of reoperative interventions, strong consideration for repeat operation should be provided to any ARM patient presenting with ongoing symptoms. Adequate examination and evaluation may demonstrate ongoing pathology that can be addressed with a repeat operation, some of which are very straightforward and can provide significant symptomatic relief.

\section{Acknowledgments}

Funding: None.

\section{Footnote}

Provenance and Peer Review: This article was commissioned by the Guest Editors (Eduardo Perez, Samir Pandya, and Matthew S. Clifton) for the series "Current Topics in Pediatric General Surgery" published in Translational Gastroenterology and Hepatology. The article has undergone external peer review.

Conflict of Interest: All authors have completed the ICMJE uniform disclosure form (available at http://dx.doi. org/10.21037/tgh-20-214). The series "Current Topics in Pediatric General Surgery" was commissioned by the editorial office without any funding or sponsorship. The authors have no other conflicts of interest to declare.

Ethical Statement: The authors are accountable for all aspects of the work in ensuring that questions related to the accuracy or integrity of any part of the work are appropriately investigated and resolved.

Open Access Statement: This is an Open Access article distributed in accordance with the Creative Commons Attribution-NonCommercial-NoDerivs 4.0 International License (CC BY-NC-ND 4.0), which permits the noncommercial replication and distribution of the article with the strict proviso that no changes or edits are made and the original work is properly cited (including links to both the formal publication through the relevant DOI and the license). See: https://creativecommons.org/licenses/by-nc-nd/4.0/.

\section{References}

1. Stephens FD. Congenital imperforated rectum, rectourethral and recto-vaginal fistulae. Aust N Z J Surg 1953;22:161-72.

2. Stephens FD. Imperforate rectum; a new surgical technique. Med J Aust 1953;1:202-3.

3. Peña A, Devries PA. Posterior sagittal anorectoplasty: Important technical considerations and new applications. J 
Pediatr Surg 1982;17:796-811.

4. deVries PA, Peña A. Posterior sagittal anorectoplasty. J Pediatr Surg 1982;17:638-43.

5. Peña A. Posterior sagittal anorectoplasty as a secondary operation for the treatment of fecal incontinence. J Pediatr Surg 1983;18:762-73.

6. Georgeson KE, Inge TH, Albanese CT. Laparoscopically assisted anorectal pull-through for high imperforate anus--a new technique. J Pediatr Surg 2000;35:927-30; discussion 930-1.

7. Nah SA, Ong CCP, Saffari SE, et al. Anorectal malformation \& Hirschsprung's disease: A cross-sectional comparison of quality of life and bowel function to healthy controls. J Pediatr Surg 2018;53:1550-4.

8. Zheng H, Liu G, Liang Z, et al. Middle-term bowel function and quality of life in low-type anorectal malformation. Ital J Pediatr 2019;45:98.

9. Kyrklund K, Pakarinen MP, Rintala RJ. Long-term bowel function, quality of life and sexual function in patients with anorectal malformations treated during the PSARP era. Semin Pediatr Surg 2017;26:336-42.

10. Divarci E, Ergun O. General complications after surgery for anorectal malformations. Pediatr Surg Int 2020;36:431-45.

11. Rintala RJ, Lindahl H. Is normal bowel function possible after repair of intermediate and high anorectal malformations? J Pediatr Surg 1995;30:491-4.

12. Chung PHY, Wong CWY, Wong KKY, et al. Assessing the long term manometric outcomes in patients with previous laparoscopic anorectoplasty (LARP) and posterior sagittal anorectoplasty (PSARP). J Pediatr Surg 2018;53:1933-6.

13. Lane VA, Skerritt C, Wood RJ, et al. A standardized approach for the assessment and treatment of internationally adopted children with a previously repaired anorectal malformation (ARM). J Pediatr Surg 2016;51:1864-70.

14. Levitt MA, Bischoff A, Peña A. Pitfalls and challenges of cloaca repair: How to reduce the need for reoperations. J Pediatr Surg 2011;46:1250-5.

15. Rosen NG, Hong AR, Soffer SZ, et al. Rectovaginal fistula: A common diagnostic error with significant consequences in girls with anorectal malformations. J Pediatr Surg 2002;37:961-5.

16. Atala A, Ellsworth P, Share J, et al. Comparison of sonicated albumin enhanced sonography to fluoroscopic and radionuclide voiding cystography for detecting vesicoureteral reflux. J Urol 1998;160:1820-2.

17. Rentea RM, Halleran DR, Vilanova-Sanchez A, et al.
Diagnosis and management of a remnant of the original fistula (ROOF) in males following surgery for anorectal malformations. J Pediatr Surg 2019;54:1988-92.

18. Ardelean MA, Bauer J, Schimke C, et al. Improvement of continence with reoperation in selected patients after surgery for anorectal malformation. Dis Colon Rectum 2009;52:112-8.

19. Peña A, Hong AR, Midulla P, et al. Reoperative surgery for anorectal anomalies. Semin Pediatr Surg 2003;12:118-23.

20. Hrabovszky Z, Dewan PA. Revision anorectoplasty in the management of anorectal anomalies. Pediatr Surg Int 2002;18:269-72.

21. Lawal TA, Reck CA, Wood RJ, et al. Use of a HeinekeMikulicz like stricturoplasty for intractable skin level anal strictures following anoplasty in children with anorectal malformations. J Pediatr Surg 2016;51:1743-5.

22. Holbrook C, Misra D, Zaparackaite I, et al. Post-operative strictures in anorectal malformation: trends over 15 years. Pediatr Surg Int 2017;33:869-73.

23. Belizon A, Levitt M, Shoshany G, et al. Rectal prolapse following posterior sagittal anorectoplasty for anorectal malformations. J Pediatr Surg 2005;40:192-6.

24. Brisighelli G, Di Cesare A, Morandi A, et al. Classification and management of rectal prolapse after anorectoplasty for anorectal malformations. Pediatr Surg Int 2014;30:783-9.

25. Alam S, Lawal TA, Peña A, et al. Acquired posterior urethral diverticulum following surgery for anorectal malformations. J Pediatr Surg 2011;46:1231-5.

26. Kraus SJ, Levitt MA, Peña A. Augmented-pressure distal colostogram: the most important diagnostic tool for planning definitive surgical repair of anorectal malformations in boys. Pediatr Radiol 2018;48:258-69.

27. Podberesky DJ, Weaver NC, Anton CG, et al. MRI of acquired posterior urethral diverticulum following surgery for anorectal malformations. Pediatr Radiol 2011;41:1139-45.

28. Alsowayan O, Almodhen F, Alshammari A. Minimally invasive surgical approach to treat posterior urethral diverticulum. Urol Ann 2015;7:273-6.

29. Symons NR, Guenther T, Gupta A, et al. Para-neorectal mucinous adenocarcinoma following childhood pullthrough procedure for imperforate anus. Colorectal Dis 2010;12:262-3.

30. Chow JS, Paltiel HJ, Padua HM, et al. Contrast-Enhanced Colosonography for the Evaluation of Children With an Imperforate Anus. J Ultrasound Med 2019;38:2777-83.

31. Fanjul M, Samuk I, Bagolan P, et al. Tethered cord in 
patients affected by anorectal malformations: a survey from the ARM-Net Consortium. Pediatr Surg Int 2017;33:849-54.

32. Thomas JC, Brock JW. Vaginal Substitution: Attempts to Create the Ideal Replacement. J Urol 2007;178:1855-9.

33. Yu DC, Grabowski MJ, Feins NR, et al. H-type rectovaginal fistula in a patient with bilateral single ectopic ureters. J Pediatr Surg 2009;44:e27-30.

34. Wood RJ, Reck-Burneo CA, Dajusta D, et al. Cloaca reconstruction: a new algorithm which considers the role

doi: $10.21037 / \operatorname{tgh}-20-214$

Cite this article as: Tirrell TF, McNamara ER, Dickie BH. Reoperative surgery in anorectal malformation patients. Transl Gastroenterol Hepatol 2021;6:43. of urethral length in determining surgical planning. J

Pediatr Surg 2018;53:90-5.

35. Palmer BW, Trojan B, Griffin K, et al. Total and partial urogenital mobilization: Focus on urinary continence. J Urol 2012;187:1422-6.

36. Stites J, Bernabé KJ, Galan D, et al. Urinary continence outcomes following vaginoplasty in patients with congenital adrenal hyperplasia. J Pediatr Urol 2017;13:38. e1-38.e7. 